\title{
CRESCIMENTO DE LACTENTES ATÉ OS 6 MESES DE IDADE ALIMENTADOS COM LEITE MATERNO E, COM LEITE ARTIFICIAL
}

\author{
GROWTH OF BREAST AND ARTIFICIALLY FED INFANTS UP TO 6 MOI OFAGE
}

Antonio A. Barros Filho', Marco A. Barbieri², José Romano Santoro ${ }^{3}$, Heloisa Bettiol²

1,2,3 Docentes, ${ }^{3}$ Aposentado dos Departamentos de: Pediatria da Faculdade de Ciências Médicas - UNICAMP ${ }^{1}$ Puericultura e Pediatria ${ }^{2,3}$ da Faculdade de Medicina de Ribeirão Preto da Universidade de São Paulo.

Correspondência: Heloisa Bettiol. Departamento de Puericultura e Pediatria da Faculdade de Medicina de Ribeirão Preto — Campus Universitário-CEP: 14048-900 - Ribeirão Preto - SP. hbettiol@fmrp.usp.br

Barros Filho AA, Barbieri MA, Santoro JR, Heloisa Bettiol H. Crescimento de lactentes até os 6 meses de idade alimentados com leite materno e, com leite artificial. Medicina, Ribeirão Preto 29: 479487 out./dez. 1996.

RESUMO: A monitorização de lactentes é extremamente importante para a prevenção de desnutrição e/ou para a detecção de problemas, cuja primeira manifestação se dá na diminuição da velocidade de crescimento. Como o tipo de aleitamento pode interferir nesse processo o objetivo deste trabalho e comparar o crescimento de lactentes alimentados com leite materno e, com leite artificial.

Foram tomados o peso e o comprimento de 365 lactentes que, ao nascer, tiveram peso maior ou igual a $2500 \mathrm{~g}$, em uma comunidade agroindustrial de São Paulo. Desses, foram separados os que passaram a receber leite artificial, antes dos 2 meses, daqueles que receberam aleitamento materno exclusivo até os 6 meses.

Foram calculados os percentis 10,50 e 90 do peso e do comprimento deitado, segundo o sexo, e gráficos são apresentados alisados pelo método $\mathrm{H} 3 \mathrm{H} 3 \mathrm{H}$ e comparados com os dados do NCHS. As crianças alimentadas com leite materno tiveram peso maior que as com leite artificial e as do NCHS. Em relação ao comprimento, tanto as crianças que receberam leite materno, quanto as que receberam leite artificial apresentam resultados sistematicamente menores que as curvas do NCHS.

UNITERMOS: Lactente Crescimento. Aleitamento Materno. Aleitamento Artificial.

\section{INTRODUÇÃO}

O seguimento do crescimento de lactentes é extremamente importante para a prevenção da desnutrição e/ou para a detecção de problemas cuja primeira manifestação se dá na redução da velocidade de crescimento $^{1}$. Dentre as recomendações para um bom crescimento, durante os primeiros meses de vida, temos: de orientar as mães sobre a importância do aleitamento materno para a manutenção da saúde do filho e a especificidade de seus nutrientes, o esforço que os profissionais de saúde devem despender para que o aleitamento materno seja mantido pelo menos até os 6 meses de vida ${ }^{2}$, incluir mensagens sobre aleitamento materno em outros programas, tais como planejamento familiar, monitorização do crescimento e terapia de reidratação oral ${ }^{3}$. Vários trabalhos ${ }^{2 / 6}$ têm demonstrado a menor morbidade entre os lactentes exclusivamente alimentados com leite materno. Com relação ao crescimento, as observações já são diver- 
gentes, havendo trabalhos que demonstram maior ganho de peso nas crianças que recebem leite humano e outros que demonstram menor ganho ${ }^{7,8}$. Muitos fatores, além do tipo de leite usado, determinam o crescimento dos lactentes ${ }^{9}$. As considerações sobre maior ou menor ganho de peso durante a fase de aleitamento materno, têm-se baseado na comparação com as chamadas curvas de referência e na qualidade e quantidade de aleitamento artificial oferecido às crianças ${ }^{10}$.

Com a intenção de estudar a influência do leite humano sobre o crescimento de lactentes nos primeiros 6 meses de vida, foi comparado, em uma população de baixo nível econômico, o crescimento de um grupo de crianças que recebeu leite materno até os 6 meses de idade com outro que foi desmamado antes de completar 2 meses. Foi examinado também o crescimento dessas crianças em comparação com as curvas do NCHS, que são as recomendadas para a monitorização do crescimento ${ }^{11}$.

\section{CASUÍSTICA E MÉTODOS}

Para estudar a influência da duração do aleitamento materno e do artificial sobre o crescimento dos lactentes de 0 a 6 meses de idade, foram analisados os dados coletados no seguimento de 365 crianças nascidas de famílias residentes no Município de Pradópolis e acompanhadas pelos médicos do Departamento de Puericultura e Pediatria da Faculdade de Medicina de Ribeirão Preto da Universidade de São Paulo (FMRPUSP), no Centro Comunitário de Assistência Médico-Infantil de Pradópolis - CECAMIP ${ }^{12}$, onde foram desenvolvidos outros estudos ${ }^{4,13,14}$. A rotina do ambulatório de pediatria foi estruturada de forma a poder prestar assistência médica contínua, com controles periódicos, às crianças de 0 a 2 anos de idade, e que permitisse também o desenvolvimento de estudos epidemiológicos e de crescimento. Este é um estudo longitudinal, prospectivo, cujas informações foram previamente definidas e que constavam especificamente da anamnese de cada criança.

À época deste levantamento, o CECAMIP se constituía, na cidade, no único serviço de atenção à criança, incluindo a prestação de assistência médica à noite, finais de semana e feriados. As crianças eram atendidas mensalmente até a idade de 6 meses, com atendimento eventual caso apresentassem alguma patologia.

Neste estudo, foi avaliado o crescimento dos lactentes de 0 a 6 meses em função da alimentação láctea que as crianças recebiam: alimentação com leite materno versus alimentação com leite de vaca ("in natura" ou industrializado). A orientação alimentar foi feita segundo as normas preconizadas pelo Departamento ${ }^{15}$. Foram analisados apenas os dados referentes às crianças nascidas com peso maior do que $2500 \mathrm{~g}$, que não apresentavam malformações congênitas e registradas no CECAMIP antes de completar 1 mês de idade.

O crescimento foi analisado pelas medidas do peso e do comprimento, tomadas por duas funcionárias padronizadas e por um docente do Departamento, responsável pelo CECAMIP, segundo as recomendações preconizadas em várias publicações ${ }^{16,17}$. Essas medidas foram feitas em todas as consultas, mas, para a elaboração das curvas e das tabelas deste estudo, foram consideradas apenas as referentes aos retornos que ocorriam em data próxima às idades mensais das crianças.

Para a categorização segundo a duração do aleitamento materno, as crianças foram divididas em 5 grupos:

Grupo A: desmame de 0 a 2 meses de idade crianças que passaram a receber, como alimento lácteo exclusivo, leite artificial, antes de completar 2 meses de idade;

Grupo B: desmame de 2 a 4 meses de idade crianças que foram desmamadas totalmente dos 2 aos 4 meses de idade;

Grupo C: desmame de 4 a 6 meses de idade crianças que foram desmamadas totalmente dos 4 aos 6 meses de idade;

Grupo D: aleitamento materno exclusivo até 6 meses - crianças que, ao completarem 6 meses de idade, continuaram recebendo exclusivamente como alimento lácteo, o leite materno;

Grupo M: aleitamento misto ao sexto mês - crianças que, aos 6 meses, estavam sendo amamentadas, mas que recebiam também suplementação com leite artificial.

No presente estudo, serão apresentados os dados referentes aos grupos A e D. Os dados são comparados com as curvas do $\mathrm{NCHS}^{11}$. As curvas foram alisadas pelo método $\mathrm{H} 3 \mathrm{H} 3 \mathrm{H}^{18}$.

\section{RESULTADOS}

Das 429 crianças inicialmente incluídas no estudo, 19 foram retiradas por nascerem com baixo peso e 7 por apresentarem malformações. Das 40 crianças 
que preencheram as exigências, 365 chegaram ao término do seguimento por 6 meses, tendo ocorrido perda de 9,4\%. A Tabela I apresenta a distribuição da população de estudo, segundo o sexo e a duração do aleitamento materno: 77 crianças receberam leite artificial antes de completar 2 meses e 125 estavam recebendo leite materno exclusivo aos 6 meses de idade.

As análises seguintes referem-se às crianças pertencentes aos grupos A (77 crianças) e D (125 crianças).
As Tabelas II, III, IV e V apresentam os percentis 10,50 e 90 para os comprímentos em centímetros e os pesos em gramas dos lactentes (meninos e meninas), segundo o tipo de aleitamento e a idade, não alisados. As figuras 1 a 4 ilustram o crescimento do comprimento e do peso alisados pelo método $\mathrm{H} 3 \mathrm{H} 3 \mathrm{H}$, segundo o sexo e o tipo de aleitamento, comparados com os dados do NCHS ${ }^{11}$.

\begin{tabular}{|c|c|c|c|}
\hline $\begin{array}{l}\text { Duração do } \\
\text { aleitamento materno }\end{array}$ & Masculino & Feminino & Total \\
\hline A & $\begin{array}{c}39 \\
(50,6)\end{array}$ & $\begin{array}{c}38 \\
(49,4)\end{array}$ & $\begin{array}{c}77 \\
(100)\end{array}$ \\
\hline B & $\begin{array}{c}26 \\
(42,6)\end{array}$ & $\begin{array}{c}35 \\
(57,4)\end{array}$ & $\begin{array}{c}61 \\
(100)\end{array}$ \\
\hline $\mathrm{C}$ & $\begin{array}{c}16 \\
(37,2)\end{array}$ & $\begin{array}{c}27 \\
(62,8)\end{array}$ & $\begin{array}{c}43 \\
(100)\end{array}$ \\
\hline $\mathrm{D}$ & $\begin{array}{c}70 \\
(56,0)\end{array}$ & $\begin{array}{c}55 \\
(44,0)\end{array}$ & $\begin{array}{l}125 \\
(100)\end{array}$ \\
\hline M & $\begin{array}{c}28 \\
(47,5)\end{array}$ & $\begin{array}{c}31 \\
(52,5)\end{array}$ & $\begin{array}{c}59 \\
(100)\end{array}$ \\
\hline TOTAL & 179 & 186 & 365 \\
\hline
\end{tabular}

$\mathrm{A}=0$ a 2meses; $\mathrm{B}=2$ a 4 meses; $\mathrm{C}=4$ a 6 meses; $\mathrm{D}=$ exclusivo até 6 meses; $\mathrm{M}$ misto ao $6^{\circ}$ mês.

Tabela II - Valores dos percentis 10, 50 e 90 do comprimento em centimetros de lactentes do sexo masculino, segundo o tipo de aleitamento e a idade.

\begin{tabular}{lrccccc}
\hline \multicolumn{7}{c}{ Idade Artificial } \\
\hline \multirow{3}{*}{ Nascimento } & P10* & P50 & P90 & P10 & P50 & P90 \\
1 mês & 48,0 & 50,0 & 51,5 & 46,0 & 49,0 & 51,0 \\
2 meses & 50,5 & 53,0 & 55,4 & 50,0 & 52,3 & 55,0 \\
3 meses & 53,4 & 56,5 & 60,0 & 52,0 & 56,0 & 59,0 \\
4 meses & 56,0 & 60,0 & 62,1 & 56,0 & 59,0 & 61,5 \\
5 meses & 58,4 & 62,0 & 65,1 & 59,0 & 62,0 & 64,5 \\
6 meses & 61,0 & 64,0 & 67,1 & 61,5 & 64,0 & 67,0 \\
\hline
\end{tabular}

$* \mathrm{P} 10=$ percentil 10, P50 $=$ percentil 50 e P90 = percentil 90. 
Tabela III - Valores dos percentis 10, 50 e 90 do comprimento em centímetros de lactentes do sexo feminino, segundo o tipo de aleitamento e a idade.

\begin{tabular}{lrccccc}
\hline \multicolumn{7}{c}{ Tipo de Aleitamento } \\
\hline Idade & \multicolumn{7}{c}{ Artificial } & \multicolumn{3}{c}{ Materno } \\
\hline \multirow{3}{*}{ Nascimento } & P1O* & P50 & P90 & P10 & P50 & P90 \\
1 mês & 46,8 & 48,0 & 50,8 & 46,1 & 49,0 & 51,0 \\
2 meses & 50,5 & 51,8 & 54,8 & 50.0 & 52,0 & 54,0 \\
3 meses & 52,0 & 54,0 & 58,6 & 53,0 & 55,0 & 58,3 \\
4 meses & 54,8 & 57,0 & 60,1 & 55,0 & 58,0 & 61,0 \\
5 meses & 57,0 & 60,0 & 62,0 & 58,0 & 60,5 & 63,0 \\
6 meses & 59,4 & 62,0 & 65,0 & 60,0 & 62,5 & 65,5 \\
\hline
\end{tabular}

*P10 $=$ percentil $10, \mathrm{P} 50=$ percentil 50 e P90 = percentil 90

Tabela IV - Valores dos percentis 10, 50 e 90 do peso em gramas de lactentes do sexo masculino, segundo o tipo de aleitamento e a idade.

\begin{tabular}{lcccccc}
\hline \multicolumn{7}{c}{ Tipo de Aleitamento } \\
\hline Idade & \multicolumn{7}{c}{ Artificial } & M90 & P10 & P50 & P90 \\
\hline \multirow{2}{*}{ Nascimento } & P10* & P50 & P90 \\
1 mês & 2890 & 3350 & 3900 & 2770 & 3400 & 4370 \\
2 meses & 3350 & 4000 & 4380 & 3540 & 4300 & 5170 \\
3 meses & 4330 & 4920 & 5600 & 4770 & 5290 & 6310 \\
4 meses & 4940 & 5780 & 6750 & 5390 & 6120 & 7250 \\
5 meses & 5710 & 6650 & 7550 & 6060 & 6840 & 8140 \\
6 meses & 6280 & 7340 & 8280 & 6410 & 7420 & 8820 \\
\hline
\end{tabular}

*P10 $=$ percentil $10, \mathrm{P} 50=$ percentil 50 e P90 $=$ percentil 90

Tabela V - Valores dos percentis 10, 50 e 90 do peso em gramas de lactentes do sexo feminino segundo o tipo de aleitamento e a idade.

\begin{tabular}{lcccrcr}
\hline Tipo de Aleitamento & \multicolumn{3}{c}{ Materno } \\
\hline Idade & \multicolumn{3}{c}{ Artificial } & P10 & P50 & P90 \\
Nascimento & 2580 & 3100 & 3350 & 2780 & 3300 & 3800 \\
1 mês & 3030 & 3670 & 3870 & 3540 & 4060 & 4570 \\
2 meses & 3910 & 5150 & 5370 & 4310 & 5030 & 5600 \\
3 meses & 4550 & 5150 & 5370 & 5630 & 5800 & 6500 \\
4 meses & 4900 & 5850 & 6150 & 5470 & 6540 & 7230 \\
5 meses & 5240 & 6550 & 6940 & 6120 & 7000 & 8040 \\
6 meses & 5480 & 6890 & 7480 & 6360 & 7470 & 8500 \\
\hline
\end{tabular}

*P10 $=$ percentil 10, P50 $=$ percentil 50 e P90 = percentil 90 
O primeiro fato que chama a atenção é o de que embora os meninos e as meninas tivessem o peso ao nascer maior que $2500 \mathrm{~g}$, tanto o grupo dos alimentados com leite materno como o grupo dos alimentados com leite artificial apresentam, sistematicamente percentis 10,50 e 90 do comprimento menores do que os respectivos percentis das curvas de referência do NCHS. As meninas, com leite materno, apresentam comprimento maior do que as com leite artificial nos 3 percentis assinalados. Embora, aos 6 meses de idade os meninos alimentados com leite humano apresentem valores similares aos dos alimentados com leite artificial, partiram do nascimento com um comprimento menor e apresentaram um "catch-up" durante os 3 primeiros meses (Figura 1). Em relação ao peso, observa-se que tanto os lactentes do sexo masculino como

\section{Masculino}
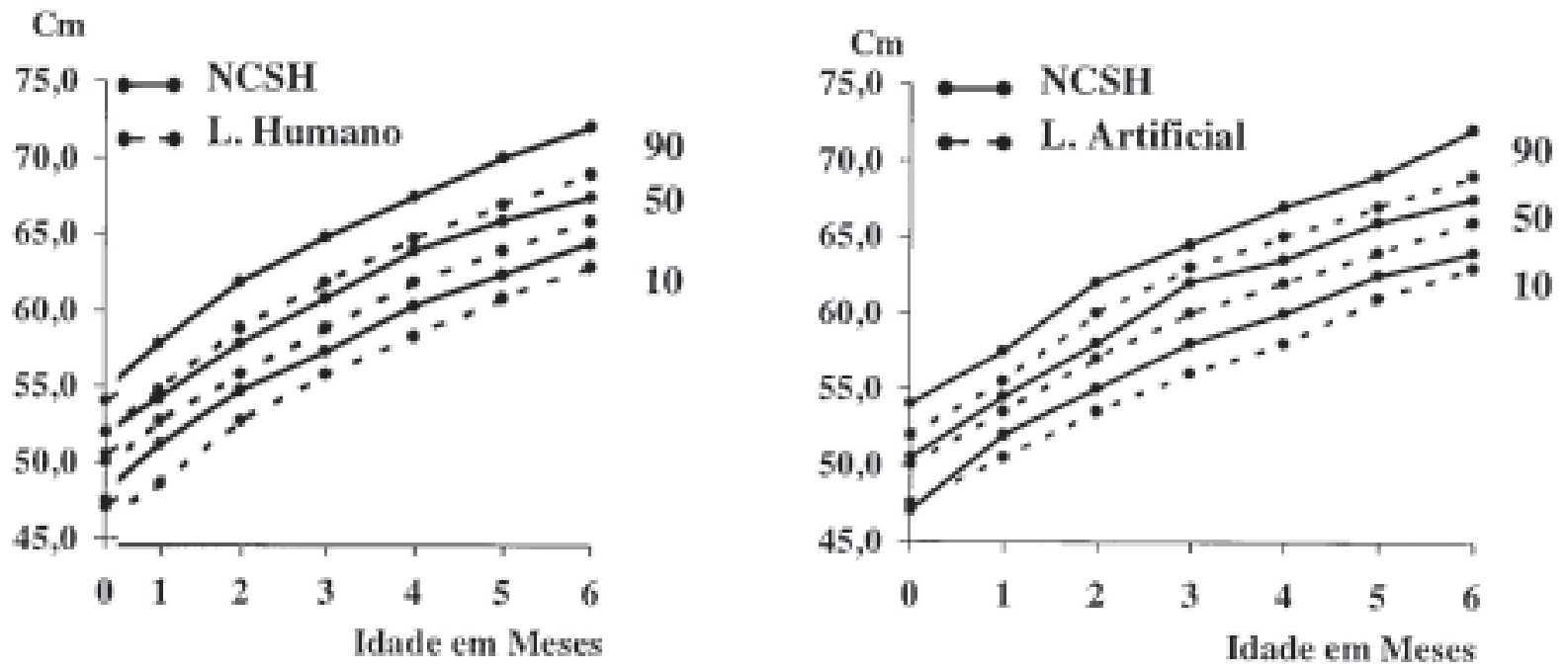

\section{Feminino}
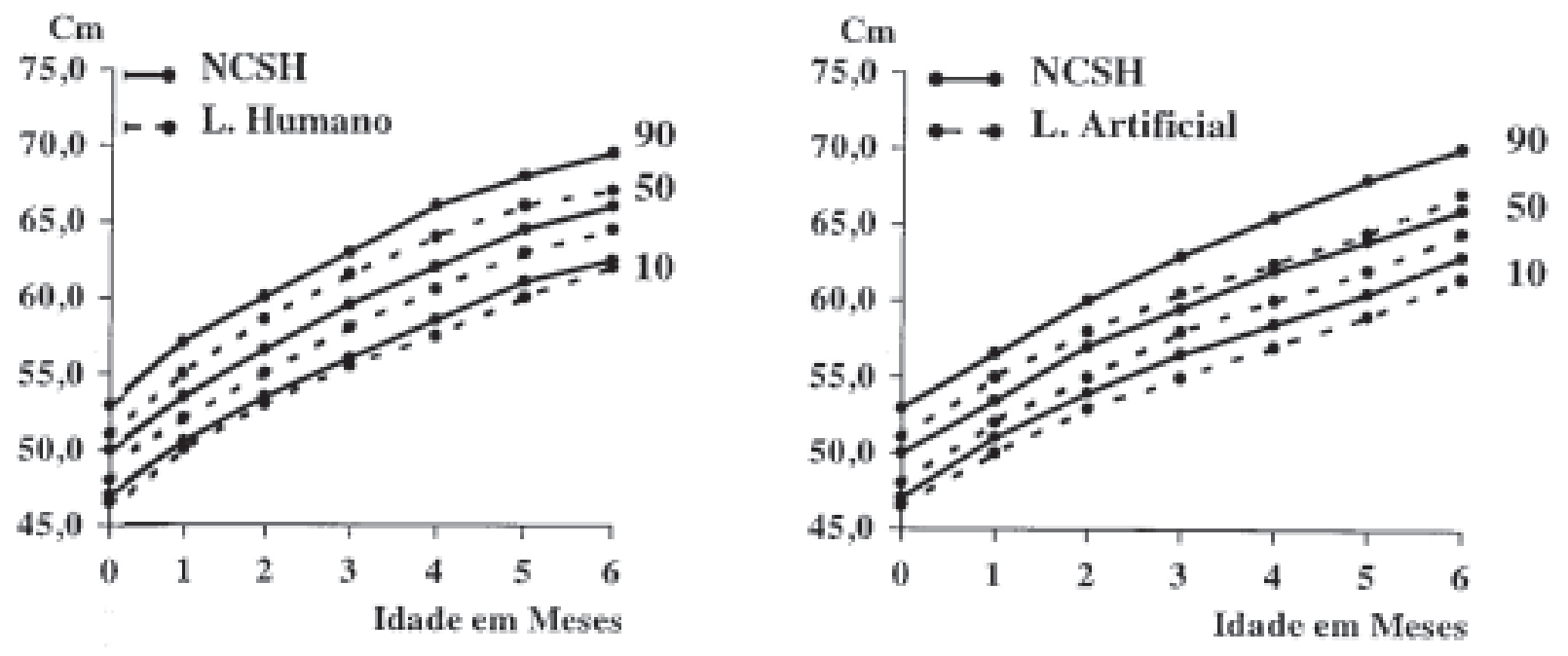

Figura 2 - Comprimento de lactentes do sexo feminino de $\mathrm{O}$ a 6 meses de idade, segundo o tipo de aleitamento, comparados com dados do NCHS. 
os do sexo feminino que receberam leite humano são mais pesados do que os que receberam leite artificial e do que os valores da curva de referência. Em relação ao aleitamento artificial, a situação não é tão favorável para ambos os sexos, mas é pior para os lac- tentes do sexo feminino, que apresentam valores do percentil 90 próximos ao percentil 50 da curva de referência e percentil 10 com tendência à queda, a partir do terceiro mês de idade.

\section{Masculino}
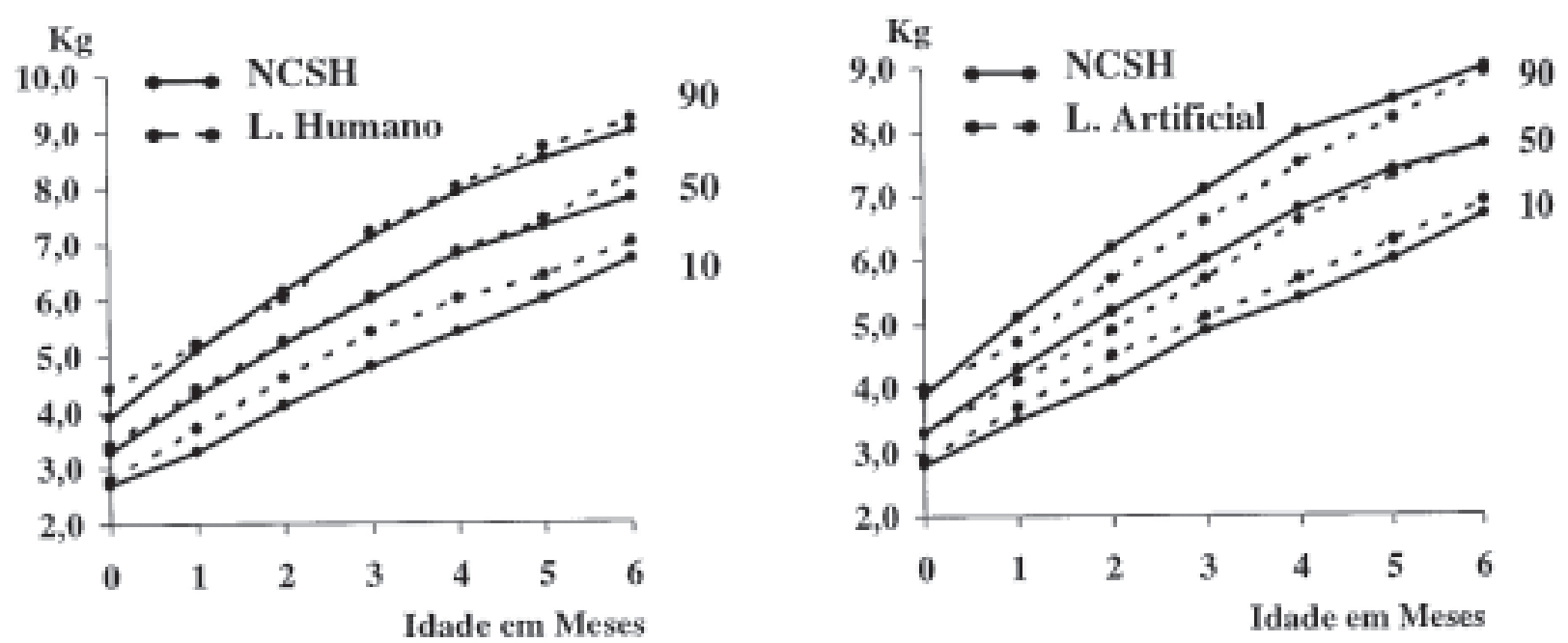

Figura 3 - Peso de lactentes do sexo masculino de O a 6 meses de idade, segundo o tipo de aleitamento, comparados com dados do NCHS.

\section{Feminino}
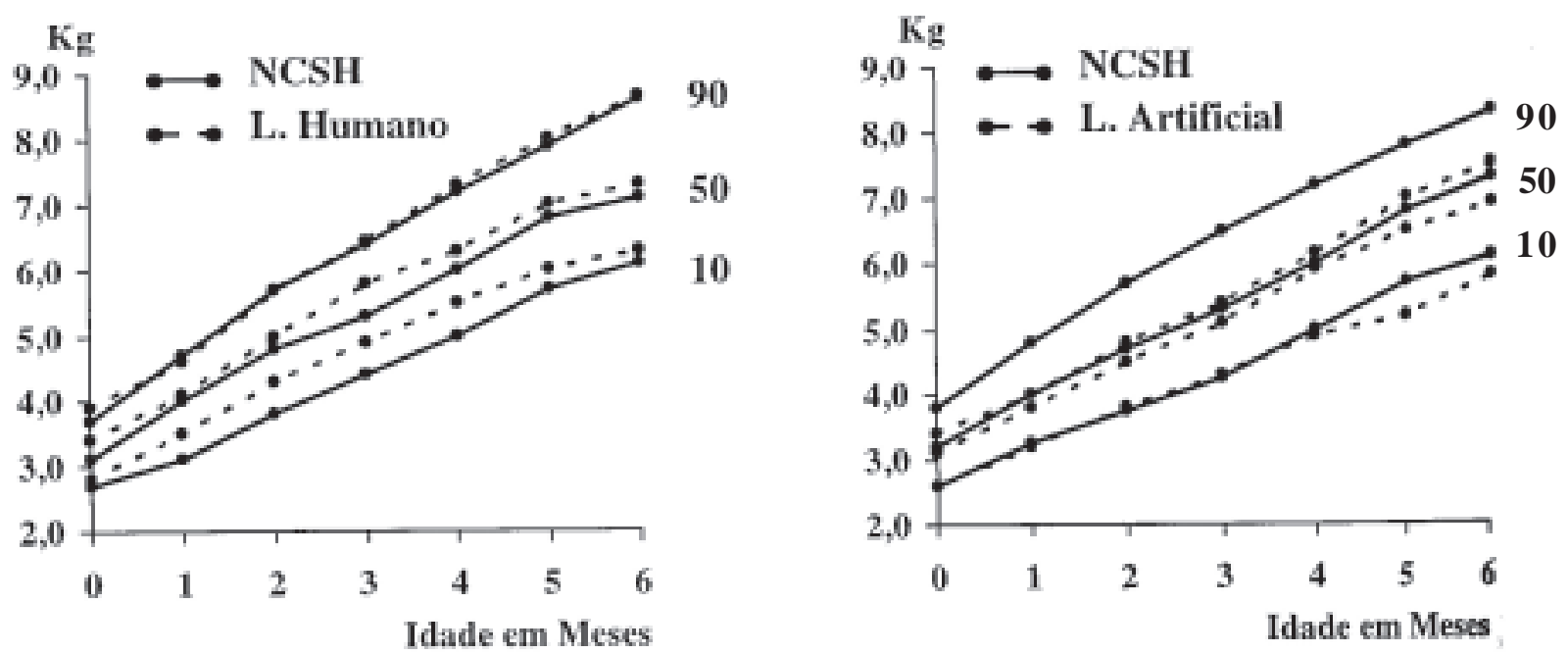

Figura 3 - Peso de lactentes do sex ofeminino de O a 6 meses de idade, segundo o tipo de aleitamento, comparados com dados do NCHS. 


\section{DISCUSSÃO}

Neste trabalho, são apresentadas as curvas de crescimento, comparando-as com os percentis 10, 50 e 90 do NCHS, complementando trabalho anterior que apresentava somente os valores medianos do peso e comprimento e as velocidades medianas do peso ${ }^{13}$.

A comparação das curvas chama a atenção para dois pontos considerados importantes:

1) o menor comprimento destes lactentes desde o nascimento até os seis meses de idade, independentemente do tipo de aleitamento;

2) o peso maior, tanto para os meninos como para as meninas que receberam leite materno até os 6 meses, tanto em relação às curvas do NCHS quanto em relação às curvas das crianças que receberam leite artificial.

Embora a literatura seja rica em estudos sobre crescimento de lactentes segundo o tipo de alimentação no primeiro ano de vida, os resultados são contraditórios ${ }^{19} \mathrm{e}$ os autores recomendam estudos longitudinais para estudar os ganhos ponderais mensais, principalmente entre os 3 e os 6 meses de vida. No Brasil são poucos os estudos relacionados ao tipo de aleitamento e crescimento ${ }^{1,13,19,20 .}$

COUTINHO et al. $1988^{20}$, estudando o crescimento de 135 crianças nascidas a termo e acompanhadas até os 12 meses de idade, concluíram que o tipo de aleitamento não teve influência sobre o crescimento, no primeiro ano de vida. MURAHOVISCHI et al. $1986^{1}$, publicaram curvas de crescimento para peso e comprimento, sem analisarem comparativamente com outro tipo de aleitamento ou referência.

Em trabalho anterior ${ }^{4}$ foi demonstrado que a mediana de ganho ponderal era de $28 \mathrm{~g} / \mathrm{dia}$, no primeiro trimenstre, e de $23 \mathrm{~g} / \mathrm{d}$, no segundo trimestre, para os meninos que recebiam leite artificial e de $22,4 \mathrm{e}$ $20 \mathrm{~g} / \mathrm{d}$, respectivamente, para as meninas alimentadas com leite artificial. Para os lactentes que recebiam leite materno, esses valores foram, respectivamente, 31 e $19 \mathrm{~g} / \mathrm{d}$ para os meninos e 26,6 e $18,5 \mathrm{~g} / \mathrm{d}$ para as meninas. O incremento mediano semestral foi de $4280 \mathrm{~g}$ para os meninos e $3640 \mathrm{~g}$ para as meninas com aleitamento artificial e $44354080 \mathrm{~g}$, respectivamente, com aleitamento materno. $\mathrm{O}$ incremento mediano semestral para o comprimento foi igual nos 4 grupos de lactentes: com leite artificial foi de $16,5 \mathrm{~cm}$ para os meninos e de $16 \mathrm{~cm}$ para as meninas, e com leite humano foi de 17 e $16 \mathrm{~cm}$, respectivamente. Como se pode depreender destes dados, o efeito protetor do leite humano em relação ao crescimento se manteve durante todo o primeiro semestre, mas se fez sentir principalmente nos 3 primeiros meses de vida, uma vez que o ganho mediano é menor no segundo trimestre. DUNCAN et al. $1984^{21}$, estudando um grupo de 33 lactentes alimentados exclusivamente ao seio até os seis meses, relatam uma menor velocidade de crescimento tanto para o peso quanto para o comprimento $\mathrm{e}$ o perímetro craniano, quando comparados com o NCHS. MARTINES et al. $1994^{22}$ estudaram crianças de famílias de baixa renda, durante 26 semanas de vida, buscando a relação entre seu padrão de alimentação e crescimento, modificado pela acesso à água e diarréia. Observaram que, nas crianças cujo alimento predominante era leite materno, o crescimento era similar em casas com e sem filtros de água; em casas sem filtros, o ganho ponderal de crianças não amamentadas ao seio era em torno da metade do observado nas crianças amamentadas, durante os primeiros 3 meses de vida; nas casas com filtros de água, o crescimento dos não amamentados era semelhante ou maior do que o das crianças alimentadas com leite materno. PATHAK et al. $1993^{23}$, observaram que o padrão de crescimento em peso, comprimento, e peso/ comprimento em lactentes até 12 meses de idade, quando comparados com a referência do NCHS, era linear nas crianças amamentadas ao seio. Neste estudo, observou-se que o peso é maior nos lactentes alimentados com leite materno, e não há diferenças quanto ao comprimento. Outro aspecto a ser comentado é que todos os percentis para as crianças de Pradópolis alimentadas com leite humano são menores do que os encontrados por MURAHOVISCHI et al. em Santos, SP, $1986^{1}$.

A população atendida no CECAMIP é de baixo nível sócio-econômico ${ }^{4,12}$ e é provável que a redução do aleitamento materno seja resultado de um peso maior ao nascer ${ }^{4,13,24}$. A tendência observada de um compri mento já afetado no prirneiro semestre de vida, e uma queda da velocidade de ganho ponderal nos dois tipos de aleitamento, no segundo trimestre, sugerem uma tendência da população se encaminhar para estaturas mais baixas já no primeiro semestre de vida, fato já encontrado por CHAVEZ \& MARTINEZ, $1979^{25} \mathrm{em}$ população rural mexicana. Para uma melhor prevenção da baixa estatura a longo prazo, sugere-se a suplementação alimentar das mães durante a gestação e lactação ${ }^{25}$ e a monitorização do crescimento dos lactentes para complementar-lhes a alimentação, no segundo trimestre, quando se desviarem da 
curva, mesmo na vigência do aleitamento materno, uma vez que tem sido descrito a respeito de crianças que não crescem de maneira apropriada mesmo quando amamentadas ${ }^{26,27,28}$. Por outro lado fica evidente, pela boa velocidade de crescimento, no primeiro trimestre, no grupo alimentado com leite materno, a importância da sua manutenção nos primeiros meses de vida.

Barros Filho AA, Barbieri MA, Santoro JR, Heloisa Bettiol H. Growth of breast and artificially fed infants up to 6 months of age. Medicina, Ribeirão Preto, 29 :479-487 oct/dec 1996.

ABSTRACT: Monitoring growth of infants is extremely important to detect malnutrition and/or ilness that the first sign is the slowing down of the rate of growth. Considering that the kind of milk provided to the children may interfere in this process this research is aimmed at comparing the growth of breast and artificially fed infants up to the age of 6 months

The weight and length of 365 infants bom with birthweight $>2500 \mathrm{~g}$ in an agro-industrial town in the State of São Paulo (Brazil) were studied. For this research we took two groups: one with children weaned before 2 months and the other with those that were breast fed up to 6 months of age.

The 10th, 50th and 90th centile for weight and length were calculated according to age and sex, and then smoothed by the $\mathrm{H} 3 \mathrm{H} 3 \mathrm{H}$ method Graphically they were compared with the NCHS reference data Children fed with breast milk showed to be heavier than the artificially fed children and the $\mathrm{NCHS}$ data. On the other hand the length of both breast fed and bottle fes infants were shorter than the NCHS

UNITERMS: Infant Growth. Breast Feeding. Bottle Feeding.

\section{REFERÊNCIAS BIBLIOGRÁFICAS}

1 - MURAHOVSCHI J et al. Curvas e tabelas decrescimento de lactentes brasileiros de 0-6 meses de idade alimentados exclusivamente com leite materno. J Pediatr, Rio de Janeiro 63:153-175,1987.

2 - JELLIFFE DB \& JELLIFFE EF. Human milk in the modern world. Oxford University Press, Oxford, 1978.

3 - PARLATO MB. The use of mass mediato promote breastfeeding. IntJ Gynaecol Obstet 3I: 105-113, 1990 Suppl 1.

4 - BARROS FILHO AA. Crescimento, morbidade e leite materno. Estudo longitudinal no Município de Pradópolis. Tese de Doutorado, Faculdade de Medicina de Ribeirão Preto da USP, Ribeirão Preto, 1981.

5 - LAWRENCE RE. Breastfeeding. A guide for the medical profession. 2 th ed, C.V. Mosby, St. Louis, 1985.

6 - HUFFMAN SL \& MARTIN L. Child nutrition, birth spacing and child mortality. Ann N Y Acad Sci 709: 236-248, 1994.

7 - OSKI FA. Infant nutrition, physical growth, breastfeeding, and general nutrition. Curr Opin Pediatr 5: 385-388, 1993.
8 - GULDAN GS et ai. Weaning practices and growth in rural Sichuan infants: a positive deviance study. J Trop Pedialr 39:168-175,1993.

9 - POSKIT EME. Practical paediatric Nutrition. Butterwooths London, 1988.

10 - WHITEHEAD RG \& PAUL, AA. Human lactation, infant feeding and growth: secular trends. In: GRACEY M \& FALKNER F. Nutritional needs and assessment of normal growth. Raven Press, New York, p. 85-122, 1985. (Nestlé Nutrition Wokshop Series, v. 7).

11 - WORLD HEALTH ORGANIZATION. Measuring change in nutritional status. Guidelines for assessing the nutritional impact of supplementary feeding programmes for vulnerable groups. WHO, Geneva, 1983.

12 - BARBIERI MA et ai. Atenção à infância em serviço comuunitário ligado à universidade. Educ Med Salud 11:5055,197

13-BARROS FILHO AA; BARBIERI, MA \& SANTORO influence of breast feeding duration on children's growth. Early Child Dev Care 15: 69-83, 1984.

14- BARROS FILHO AA; BARBIERI MA; SANTORO JR. Influência da duração do aleitamento materno na morbidade de lactentes. Bol Of Sanit Panam 64: 594-604, 1985. 
15 - WOISKI JR. Dietética pediátrica. Livraria Atheneu, Rio de Janeiro 1981.

16 - FALKNER F. Office measurement of physical growth. Pediatr Clin North Am 8:13-18, 1961.

17 - JELLIFFE DB. The assessment of the nutritional status of the community (with special reference to field surveys in developing regions of the world). WHO Monograph Ser 53: 3$271,1966$.

18 - DACHS N. Microtab: Sistema para análise estatística. Optima, Campinas, s/d.

19 - RICCO RG. Crescimento segundo o tipo de aleitamento, de crianças de um a seis meses de idade, atendidas no Centro Medico Social de Vila Lobato. Tese de Livre-Docência de Medicina de Ribeirão Preto da USP, Ribeirão, 1987.

20 - COUTINHO SB et al. influência do tipo de aleitamento sobre o ganho pondo-estatural de crianças no primeiro ano de vida. Rio de Janeiro 64: 75-82, 1988.

21 - DUNCAN B; SCHAEFFER C; SIBLEY B; FONSECA NM. Reduced growth velocity in exclusively breast-fed infants. Am.J Dis Child 138: 309-313, 1984.
22 - MARTINES JC et aL. Weaning in southern Brazii: is there a "weanlings dilemma"?. J Nutr 124: 1189-1198, 1994.

23 - PATHAK A; SHAH N \& TATARI1A A. Growth of exclusively breastfed infants. Indian Pediatr 30: 1291-1300, 1993.

24 - BARROS FC et al. Birth weight and duration of brestfeeding: DB. are the beneficial effects of human miik being overestimated? Pediatrics 78:656-661, 1986.

25 - CHAVEZ A \& MART1NEZ C. Nutrición y desarroilo infantil. Interamericana, México, 1979.

26 - EVANS TJ \& DAVI1ES DP. Failure to thrive at the breast: an old problem revisited. Arch Dis Child 51: 974-975, 1977.

27 - WATERLOW JC \& THOMSON AM. Observations on the adequacy of breast-feeding. Lancet 2: 238-242, 1979.

28 - WATERLOW JC. Reflections on stunting. Int Child Health 2:25-35, 1995

Recebido para publicação em 03/07/96

Aprovado para publicação em 31/10/96 